\title{
The use of histological techniques for the demonstration of ion exchange resins
}

\author{
A J Chaplin
}

\begin{abstract}
Aim-To establish the staining characteristics of certain ion exchange resins in histological material, with a view to enabling confident differential identification.

Methods-Various histological staining procedures were applied to selected pathological material and prepared agar blocks containing the cation exchange resin calcium polystyrene sulphonate and the anion exchange resin cholestyramine. Results-Calcium polystyrene sulphonate uniquely stained strongly by a direct Schiff's reagent procedure without any preoxidation and by the Ziehl-Neelsen method. Cholestyramine was negative by the former method but stained strongly with a standard Congo red technique.

Conclusions-These staining results are consistent with the known structure and properties of polystyrene sulphonate and cholestyramine resins. Polystyrene sulphonate resins have the virtually pathognomonic feature of direct Schiff positivity, while morphology, location, and strong non-birefringent Congo red positivity facilitate the identification of cholestyramine. It is possible that the intrinsic staining characteristics of cholestyramine may be lost once it has bound to its target. (f Clin Pathol 1999;52:776-779)
\end{abstract}

Keywords: ion-exchange resin; polystyrene sulphonate; cholestyramine

Accepted for publication 24 May 1999

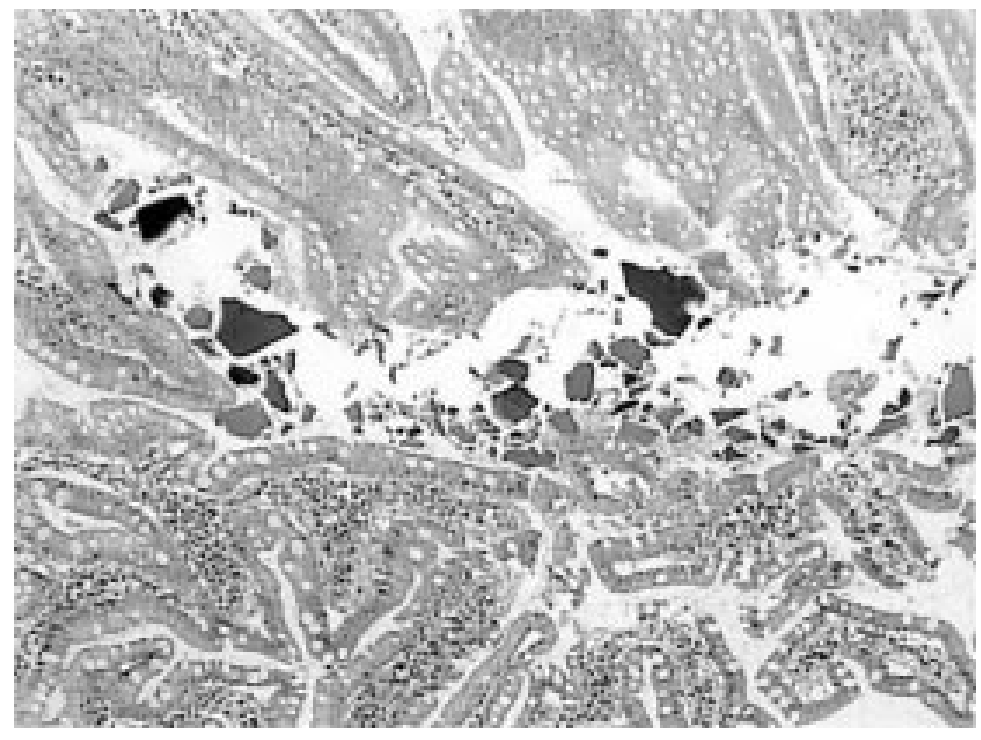

Figure 1 Incidental luminal polystyrene sulphonate resin particles in jejunal diverticular tissue. Haematoxylin and eosin, $\times 120$.
Sodium polystyrene sulphonate (Resonium A, Kayexalate) and calcium polystyrene sulphonate (Calcium Resonium, KayexalateCalcium) are cation exchange resins given orally or by retention enema for the treatment of hyperkalaemia associated with anuric and severe oliguric renal insufficiency. They may also be used to treat hyperkalaemia in patients requiring dialysis, including those on regular haemodialysis or prolonged peritoneal dialysis. Chemically, the resins are sulphonated cross linked styrene divinylbenzene copolymers.

A brief report from this laboratory in 1975 first described a picture of aspiration pneumonia as a sequel to the administration of Calcium Resonium in an elderly debilitated patient. ${ }^{1}$ Since this initial report, cation exchange resins have been implicated in contributing to respiratory problems in infants ${ }^{2}$ as well as in adults, ${ }^{34}$ and also in gastrointestinal complications subsequent to use of the resins in the treatment of hyperkalaemia in neonates, ${ }^{5}{ }^{6}$ following renal transplantation, ${ }^{7}$ and in other renal disease. ${ }^{89}$ In cases of colonic necrosis linked to the use of polystyrene sulphonates, the resin is regarded as a bystander and of lesser significance than the sorbitol solution in which it is frequently given. ${ }^{8}$ Indeed, the resin particles may be encountered histologically from time to time as strongly basophilic, angular particles, adherent to the otherwise unremarkable or uninvolved gastrointestinal mucosa of patients who have received the resin without ill effect.

A recent article, reporting two cases of sodium polystyrene sulphonate aspiration in children, described the use of infrared microspectrophotometry to confirm the identity of the resin particles seen in alveoli. ${ }^{2}$ Such techniques are costly and not immediately available to many pathologists. Simple but reliable histological identification may therefore be preferable. ${ }^{10}$

Cholestyramine (Questran) is an anion exchange resin used in the management of hypercholesterolaemia, and to control diarrhoea associated with Crohn's disease, ileal resection, vagotomy, or diabetic neuropathy. Cholestyramine is cross linked trimethylammonium benzyl chloride, the cross linking agent being a divinylbenzene styrene polymer. It acts by binding bile acids, preventing their reabsorption, so in turn promoting hepatic conversion of cholesterol into bile acids and reducing plasma low density lipoprotein cholesterol levels.

There are apparently no published reports in which any adverse affects attributed to cholestyramine involve histological manifestation of 
Table 1 Summary of staining properties of ion exchange resins

\begin{tabular}{lllll}
\hline & $\begin{array}{l}\text { Polystyrene sulphonate } \\
\text { (in agar or tissue) }\end{array}$ & & $\begin{array}{l}\text { Cholestyramine } \\
\text { (in agar) }\end{array}$ \\
\hline Unstained & $\begin{array}{l}\text { Weakly refractile } \\
\text { Colourless } \\
\text { Weakly birefringent }\end{array}$ & & $\begin{array}{l}\text { Not refractile } \\
\text { Colourless }\end{array}$ \\
H\&E & Deep violet blue & +++ & $\begin{array}{l}\text { Very weakly birefringent } \\
\text { Orange red / magenta }\end{array}$ & ++++ \\
Schiff (unoxidised) & Magenta & ++++ & Unstained & - \\
Periodic acid Schiff & Magenta & +++++ & Pink & ++ \\
Ziehl-Neelsen & Magenta & ++++ & Unstained & - \\
Van Gieson & Pink & ++ & Bright orange red & +++ \\
Orcein & Deep purple black & ++++ & Violet & +++ \\
Alcian blue pH 2.5 & Unstained & - & Unstained & - \\
Azo eosin & Dark red deposit & ++++ & Bright red & ++++ \\
Congo red & Unstained & - & Bright vermillion & ++++ \\
Giemsa & Dark blue black & +++++ & Orange / brown black & ++++ \\
\hline
\end{tabular}

*The dye appeared as a dense deposit on and around the resin particles which were themselves apparently unstained.

$\mathrm{H} \& \mathrm{E}$, haematoxylin and eosin.

the resin, though as with polystyrene sulphonates, it may be seen incidentally in bowel specimens. ${ }^{9}$ It has been implied that in such situations, cholestyramine may possibly be confused with polystyrene sulphonates,

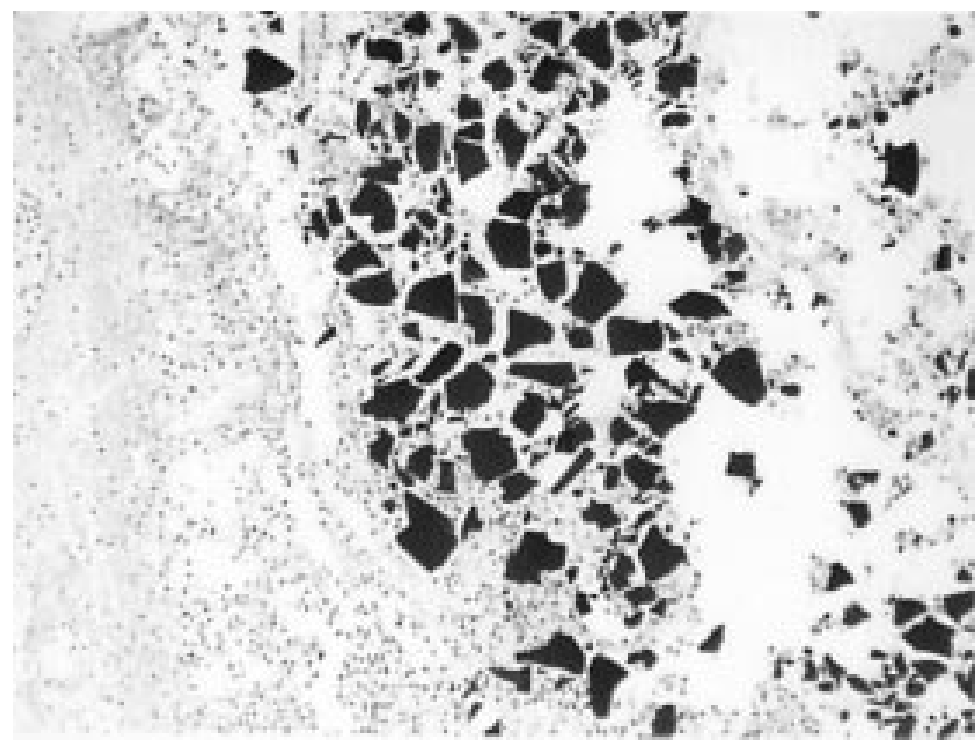

Figure 2 Polystyrene sulphonate resin particles at site of colonic necrosis. Direct Schiff with light counterstain, $\times 120$.

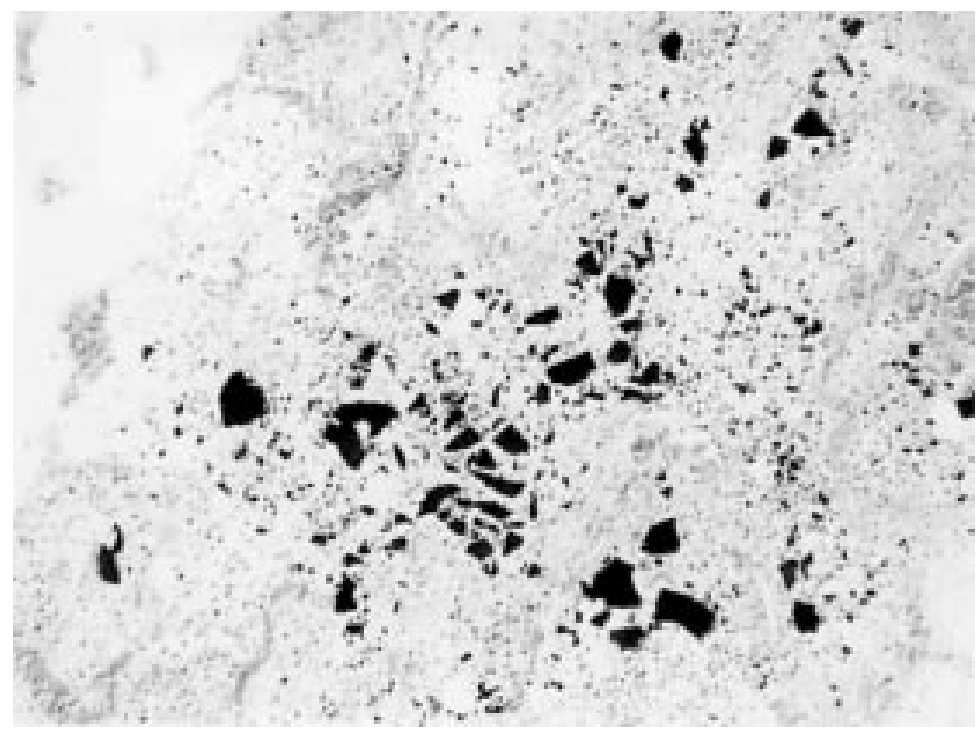

Figure 3 Polystyrene sulphonate resin particles in area of aspiration pneumonia. Ziehl-Neelsen with light counterstain, $\times 120$. though its chemical structure and function may presuppose differing histological properties. The object of this brief study was to compare the staining characteristics of polystyrene sulphonates and cholestyramine in an attempt to establish clear histological distinction.

\section{Methods}

In order to simulate regular histological material for positive control, aqueous suspensions of pure Calcium Resonium and Questran were embedded in $2 \%$ agar in saline, then fixed in $10 \%$ formol saline for 24 hours before routine overnight processing to paraffin wax through alcohol and xylene. Sections were then prepared as for normal histological material and examined unstained or stained with the following procedures: haematoxylin and eosin, Schiff's reagent (no pretreatment, no nuclear stain); periodic acid-Schiff (PAS) method (no nuclear stain); Ziehl-Neelsen (ZN) method (no nuclear stain); van Gieson's stain (no nuclear stain); orcein (with and without preoxidation with potassium permanganate); alcian blue $\mathrm{pH}$ 2.5 (no nuclear stain); azo eosin (no nuclear stain); Congo red (Puchtler and Sweat method for amyloid, no nuclear stain), and Giemsa's method. Sections from histological material shown previously to contain polystyrene sulphonates were similarly prepared and stained. This material included postmortem lung tissue from an elderly man who died of atherosclerotic heart disease, surgically removed jejunal diverticular tissue from a 74 year old woman, and a left hemicolectomy specimen obtained from a 54 year old woman after bilateral nephrectomy. (For photographic purposes, some sections were stained with Schiff's reagent followed by a light haematoxylin counterstain, and by the $\mathrm{ZN}$ method using light application of the traditional methylene blue counterstain.) Haematoxylin and eosin stained sections of gastrointestinal specimens received from 29 patients who had been prescribed cholestyramine around the time of their surgical procedure were examined. These surgical specimens had all been fixed in formol saline and processed as above.

\section{Results}

Grossly, both resins have a very pale tan appearance: this was not evident microscopically, and neither substance had any striking optical characteristic. With haematoxylin and eosin, the polystyrene sulphonates in agar blocks and tissue samples were strikingly basophilic (fig 1), while cholestyramine (in agar) was unequivocally eosinophilic. Both resins appeared as irregular, angular fragments of similar morphology, but particles of cholestyramine ( 1 to $150 \mu \mathrm{m}$ diameter) were frequently larger than those of Calcium Resonium (1 to $75 \mu \mathrm{m}$ diameter). Results with the other staining procedures are summarised in table 1 . Conspicuous among these was the direct (unoxidised) Schiff positivity and acid fast $\mathrm{ZN}$ staining of Calcium Resonium (figs 2 and 3), and the positive Congo red staining of cholestyramine (fig 4), coupled with corresponding negative staining of the other resin in each case. 


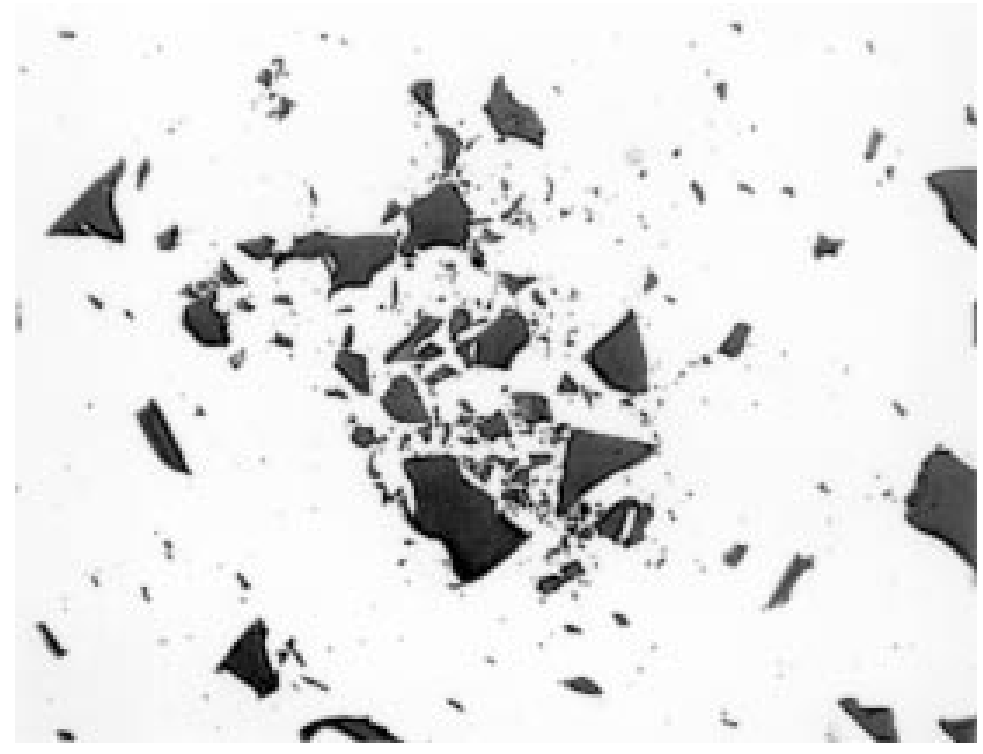

Figure 4 Cholestyramine resin particles in agar model. Congo red (Puchtler and Sweat method, no counterstain, $\times 120$ ).

Although cholestyramine was strongly positive with Congo red, there was no detectable apple green birefringence or dichroism. Staining results with polystyrene sulphonates in tissue samples were the same as in the agar blocks. None of the surgical material examined for cholestyramine showed any conclusive evidence of residual particulate matter.

\section{Discussion}

The uniqueness of direct Schiff positivity of the Resonium resins, a phenomenon first described in an histopathological context by Liber, ${ }^{11}$ was reaffirmed by these results. None of the endogenous substances known to be capable of direct reaction with Schiff's reagent is likely to be found to any substantial degree in formalin fixed, paraffin wax embedded tissue. ${ }^{12}$ It has been shown previously that direct Schiff positivity is a property shared by both sodium and calcium polystyrene sulphonates, and is attributed to their many sulphonate groupings. ${ }^{10}$ These latter also account for the intense basophilia displayed by such substances, and it is this characteristic that invariably first draws attention to the resin particles in gastrointestinal histological material. They may not be limited only to luminal or mucosal situations: there is some experimental evidence that finely ground resin particles $(1-5 \mu \mathrm{m})$ can be absorbed from the intestinal lumen and reach the lungs, brain, and lymph nodes. ${ }^{13}$ Liber noted in passing that polystyrene resin particles may become extravasated, ${ }^{11}$ and Rashid and Hamilton described in more detail one case in their series with peritoneal deposits. ${ }^{9}$ Thus the presence of such particles distant from the bowel should not necessarily be regarded as histological contamination.

In contrast to the dramatic reaction of the polystyrene sulphonates, cholestyramine remained unstained or only weakly stained by Schiff's reagent and the PAS and $\mathrm{ZN}$ methods. As it is an anion exchange resin, cholestyramine would be expected to have affinity for acid rather than basic dyes, and this was indeed confirmed by the results obtained. Positive staining with eosin and another typical acid dye, azo eosin, together with the reaction with van Gieson and Giemsa stains, were all consistent with this particular characteristic. The results are at variance with those obtained by Rashid and Hamilton, who reported that cholestyramine crystals were "more intensely basophilic . . . compared with Kayexalate crystals". ${ }^{9}$ This is a finding out of character with the anticipated histological properties of cholestyramine, and is all the more curious as the same workers also noted that cholestyramine crystals were bright orange red (that is, acidophilic) rather than basophilic in endoscopic biopsies fixed in formalin that contained eosin.

One reason for this apparent variability could be that the tinctorial characteristics of cholestyramine may alter significantly once it has bound to a suitable substrate. Bile pigments are basophilic, ${ }^{12}$ and if bound to cholestyramine may thus obscure the intrinsic staining characteristics of the resin itself or prevent staining altogether. This being the case, the apparent staining reactions of cholestyramine in situ could vary according to its functional status. The sequestration of bile acids being a complex and not necessarily irreversible process, involving hydrophobic as well as ionic attraction and retention, ${ }^{14}$ this may not be entirely predictable, but would generally relate to the point the cholestyramine had reached in its passage through the gastrointestinal tract. Such a possibility could not be substantiated in the present limited study owing to the unavailability of suitable material. It should be noted by comparison that there is no discernible difference between the histological characteristics of "pure" polystyrene sulphonates (in agar) and the equivalent resin particles found in situ, whether lung or bowel. This is not inconsistent with the suggestion above, however: once bound, a simple "invisible" cation could reasonably be expected to have a less obvious histological effect than a complex and highly pigmented anion.

In this study, it was the positive staining of cholestyramine with the acid dye Congo red that provided, in stark contrast to the negative reaction of polystyrene sulphonates, the most selective demonstration of this material. Congo red has been used as a general contrast stain with haematoxylin, but at present its principal histological use is for amyloid staining. ${ }^{15}$ As a direct cotton dye, its staining of amyloid, as of cellulose, most probably depends on a mechanism involving linear hydrogen bonding, and under the staining conditions of the Congo red technique used in this study the regular polymeric structure of cholestyramine may well favour a similar mechanism, rather than the direct coulombic attraction often involved in acid dye-tissue base interactions.

In normal tissues, only elastic fibres and eosinophil granules are likely to stain with Congo red under the conditions of the Puchtler and Sweat modification. When present, vegetable tissue fragments (for example, those from 
food residues), cotton fibres (such as contaminants from surgical dressings), and amyloid all stain strongly, but thus stained display a distinctive green birefringence. ${ }^{16}$ There should therefore be adequate distinction between non-birefringent Congophilic cholestyramine particles and luminal birefringent Congophilic vegetable tissue fragments in appropriately stained preparations. However, the influence of any complexed bile pigment must also be considered, as, if present, it could well obscure Congo red positivity.

There is no immediate explanation for the curious effect seen after the staining of Calcium Resonium with azo eosin. The resin particles themselves when visible seemed to be unstained: generally they were completely surrounded by a dense aggregation of dark red needle-like crystals in a manner reminiscent of some of the less commonly used methods for the histochemical demonstration of calcium salts, such as chloranilic acid. ${ }^{17}$ The phenomenon was observed in sections from both the agar and tissue blocks but was seen only in relation to Calcium Resonium particles: staining of cholestyramine was strong and homogeneous.

I thank Dr Bryan Warren for helpful advice, Dr M-O Peny (Université Libre de Bruxelles) for permission to use material from the case of colonic necrosis, and Karen Webber for technical support.

1 Chaplin AJ, Millard PR. Calcium polystyrene sulphonate: an unusual cause of inhalation pneumonia. $B M f$ 1975;3:77-8.
2 Fenton JJ, Johnson FB, Przygodzki RM, et al. Sodium polystyrene sulphonate (Kayexalate) aspiration. Histologic appearance and infrared microspectrophotometric analysis of two cases. Arch Pathol Lab Med 1996;120:967-9.

3 Oi RH. The microscopic appearance of a sodium potassium exchange resin in histologic sections. Am f Clin Pathol 1978;69:359-61.

4 Haupt HM, Hutchins GM. Sodium polystyrene sulphonate pneumonitis. Arch Intern Med 1982;142:379-81.

5 Metlay LA, Klionsky BL. An unusual gastric bezoar in a newborn: polystyrene resin and Candida albicans. 7 Pediatr newborn: polystyren
1983;102:121-3.

6 Ohlsson A, Hosking M. Complications following oral administration of exchange resins in extremely low-birthweight infants. Eur F Pediatr 1987;146:571-4.

7 Scott TR, Graham SM, Schweitzer EJ, et al. Colonic necrosis following SPS (Kayexalate $($ ) - sorbitol enemas in a renal transplant patient. Report of a case and review of the literature. Dis Colon Rectum 1993;36:607-9.

8 Lillemoe KD, Romolo JL, Hamilton SR, et al. Intestinal necrosis due to sodium polystyrene (Kayexalate) in sorbitol enemas: clinical and experimental support for the hypothenemas: clinical and experimental

9 Rashid A, Hamilton SR. Necrosis of the gastrointestinal tract in uremic patients as a result of sodium polystyrene sulphonate (Kayexalate) in sorbitol. Am F Surg Pathol 1997;21:60-9.

10 Chaplin AJ, Kemp P. Polystyrene sulphonates and aspiration pneumonia: a histochemical study. Histopathology 1984;8: 331-8.

11 Liber AF. The direct Schiff reaction of sodium polystyrene sulphonate (SPS), a potassium absorbing resin [abstract]. Am $\mathcal{F}$ Pathol 1974;74:106a.

12 Pearse AGE. Histochemistry theoretical and applied, vol 2: Analytical technology (4th ed). Edinburgh: Churchill Livingstone, 1985

13 Payne JM, Sansom BF, Garner RJ, et al. Uptake of small resin particles (1-5 $\mu \mathrm{m}$ diameter) by the alimentary canal of the calf. Nature 1960;188:586-7.

14 Mandeville WH, Goldberg DI. The sequestration of bile acids, a non-absorbed method for cholesterol reduction. A review. Current Pharmaceutical Design 1997;3:15-28.

15 Lillie RD. H f Conn's biological stains, 9th ed. Baltimore: Williams and Wilkins, 1977 .

16 DeLellis RL, Bowling MC. The use of Sirius red and Congo red in routine histopathology. Hum Pathol 1970;1:655.

17 Chaplin AJ, Grace SR. An evaluation of some complexing methods for the histochemistry of calcium. Histochemistry $1976 ; 47: 263-9$ 